Erratum

\title{
Mixing of pentaquark and molecular states
}

Xiao-Gang $\mathrm{He}^{1,2}$, Xue-Qian $\mathrm{Li}^{1}$, Xiang Liu ${ }^{1, \mathrm{a}}$, Xiao-Qiang Zeng ${ }^{1}$

1 Department of Physics, Nankai University, Tianjin, 300071, P.R. China

2 NCTS and Department of Physics, National Taiwan University, Taipei, 1067, Taiwan, R.O.C.

Published online: 20 October 2005 - (C) Springer-Verlag / Società Italiana di Fisica 2005

Due to an unfortunate error the paper was published online first under the wrong category.

\footnotetext{
a e-mail: lx032278@phys.nankai.edu.cn
} 